University of Nebraska - Lincoln

DigitalCommons@University of Nebraska - Lincoln

2006

Differences between Tethered Polyelectrolyte Chains on Bare

Mica and Hydrophobically Modified Mica

Marc Balastre

Phillip Schorr

J.-F. Argillier

Jinchuan Yang

Jimmy W. Mays

See next page for additional authors

Follow this and additional works at: https://digitalcommons.unl.edu/usdoepub

Part of the Bioresource and Agricultural Engineering Commons

Balastre, Marc; Schorr, Phillip; Argillier, J.-F.; Yang, Jinchuan; Mays, Jimmy W.; and Tirrell, Matthew, "Differences between Tethered Polyelectrolyte Chains on Bare Mica and Hydrophobically Modified Mica" (2006). US Department of Energy Publications. 60.

https://digitalcommons.unl.edu/usdoepub/60

This Article is brought to you for free and open access by the U.S. Department of Energy at DigitalCommons@University of Nebraska - Lincoln. It has been accepted for inclusion in US Department of Energy Publications by an authorized administrator of DigitalCommons@University of Nebraska - Lincoln. 


\section{Authors}

Marc Balastre, Phillip Schorr, J.-F. Argillier, Jinchuan Yang, Jimmy W. Mays, and Matthew Tirrell 


\title{
Differences between Tethered Polyelectrolyte Chains on Bare Mica and Hydrophobically Modified Mica
}

\author{
Feng Li,${ }^{\dagger}$ Marc Balastre, ${ }^{\dagger}$ Phillip Schorr, ${ }^{\dagger}$ J.-F. Argillier, ${ }^{\ddagger}$ Jinchuan Yang, ${ }^{\S}$ \\ Jimmy W. Mays, ${ }^{\S, f}$ and Matthew Tirrell ${ }^{*, \dagger}$ \\ Department of Chemical Engineering and the Materials Research Laboratory, University of California at \\ Santa Barbara, Santa Barbara, California 93106, Institut Français du Pétrole, 1-4 Avenue Du Bois Préau, \\ 92582 Rueil Malmaison Cedex, France, Department of Chemistry, University of Tennessee, \\ Knoxville, Tennessee 37996, and Chemical Sciences Division, Oak Ridge National Laboratory, \\ Oak Ridge, Tennessee 37831
}

Received August 23, 2005. In Final Form: January 17, 2006

\begin{abstract}
This study investigates the structures of layers of amphiphilic diblock copolymers of poly( $t$-butyl styrene $)-$ poly(styrene sulfonate) (PtBS-PSS) adsorbed on both the bare mica surface (hydrophilic) and an octadecyltriethoxysilane (OTE)-modified mica surface (hydrophobic). When the surface is rendered hydrophobic, the nonsoluble block exhibits stronger interaction with the surface and higher adsorbed masses are achieved. Interaction forces between two such adsorbed layers on both substrates were measured using the surface forces apparatus. The effect of salt concentration $\left(C_{\mathrm{s}}\right)$ and molecular weight $(N)$ on the height of the self-assembled layers $\left(L_{0}\right)$ was examined in each case. The resulting scaling relationship is in good agreement with predictions of the brush model, $L_{0} \propto N^{1.0}$ in the low-salt limit and $L_{0} N^{-1} \propto\left(C_{\mathrm{s}} / \sigma\right)^{-0.32}$ in the salted regime, when adsorption takes place onto the hydrophobized mica surface. For adsorption on the bare mica surface, $L_{0} N^{-0.7} \propto C_{\mathrm{s}}^{-0.17}$ agrees with the scaling prediction of the sparse tethering model. The results suggest that, on the hydrophilic bare mica surface, the adsorbed amount is not high enough to form a brush structure and only very little intermolecular stretching of the tethered chains occurs; in contrast, the presence of the hydrophobic OTE layer increases the tethering density such that the polyelectrolyte chains adopt a brush conformation.
\end{abstract}

\section{Introduction}

Polyelectrolytes at interfaces are of tremendous technological importance. Through the long-ranged electrostatic interactions and short-ranged van der Waals and steric interactions, polyelectrolyte chains attached to a surface offer sophisticated control over interfacial properties such as interfacial tension, lubrication, adhesion, friction, and wetting behavior. ${ }^{1-3}$ Moreover, polyelectrolytes are water-soluble and can manipulate interfacial properties in aqueous media, which makes them potentially useful in biological and environmentally friendly applications. ${ }^{4}$ It is well known that the adsorption of polymers onto colloidal particles can cause profound changes in colloidal stability. ${ }^{5,6}$ On one hand, attaching polyelectrolytes on to particle surfaces leads to a significant increase in stability through a combination of steric and electrostatic repulsion. On the other hand, the same polyelectrolytes, which provide steric and electrostatic protection when effectively adsorbed to the particle, may promote flocculation when adsorbed at a low tethering density, generating an aggregation structure. ${ }^{2,3}$ Hence, the functionality and properties of the tethered polyelectrolyte are strongly dependent on tethering density of polymer chains.

* To whom correspondence should be addressed. E-mail: tirrell@ engineering.ucsb.edu.

$\dagger$ University of California at Santa Barbara.

¥ Institut Français du Pétrole.

$\S$ University of Tennessee.

${ }^{£}$ Oak Ridge National Laboratory.

(1) Napper, D. H. Polymeric Stabilization of Colloidal Dispersions; Academic Press: London, 1985.

(2) Finch, C. A. Chemistry and Technology of Water-Soluble Polymers; Plenum Publishing Corporation: New York, London.

(3) Dautzenbrg, H.; Jaeger, W.; Kötz, J.; Philipp, B.; Seidel, C.; Stscherbina, D. Polyelectrolytes: Formation, Characterization and Application; Hanser Gardner Publications: New York, 1994

(4) Hammer, D.; Tirrell, M. Аnпu. Res. Mater. Sci. 1996, 26, 651

(5) Patel, S. S.; Tirrell, M. Annu. Rev. Phys. Chem. 1989, 40, 597.
The mechanisms available to tether the polyelectrolyte chains to surfaces and interfaces vary on the basis of the nature of the tethering site. Relevant to the solid/liquid interface are selfassembly through adsorption of the anchoring blocks of amphiphilic diblock copolymers, ${ }^{7-12}$ covalent linking of polyelectrolyte chains to a substrate, ${ }^{13-19}$ Langmuir-Blodgett (LB) deposition, ${ }^{20-22}$ and spin-coating. ${ }^{23}$

Self-assembly via adsorption of anchoring segments is a particularly simple and convenient means of creating polyelectrolyte layers tethered to a surface. In this work, amphiphilic diblock copolymers, which consist of a neutral block and a charged block, have been adsorbed to surfaces from aqueous solutions. In this case, the neutral block acts as an anchor to the surface,

(6) Stokes, R. J.; Evans, D. F. Fundamentals of Interfacial Engineering; John Wiley and Sons: New York, 1997.

(7) Dan, N.; Tirrell, M. Macromolecules 1993, 26, 4310.

(8) Amiel, C.; Sikka, M.; Schneider, J. W.; Tsao, Y.; Tirrell, M.; Mays, J. W. Macromolecules 1995, 28, 3125.

(9) Zhang, Y.; Tirrell, M.; Mays, J. W. Macromolecules 1996, 29, 7299.

(10) Zhang, Y.; Tirrell, M.; Mays, J. W. Rev. Inst. Fr. Pet. 1997, 52, 177.

(11) Kelley, T. W.; Schorr, P. A.; Johnson, K. D.; Tirrell, M.; Frisbie, C. D. Macromolecules 1998, 31, 4297.

(12) Kampf, N.; Gohy, J.; Jerome, R.; Klein, J. J. Polym. Sci., Part B: Polym. Phys. 2005, 43, 193.

(13) Mir, Y.; Auroy, P.; Auvray, L. Phys. Rev. Lett. 1995, 75, 2863.

(14) Jordan, R.; Graf, K.; Riegler, H.; Unger, K. K. Chem. Commun. 1996, 9, 1025 .

(15) Hayashi, S.; Handa, S.; Tsubokawa, N. J. Polym. Sci.: Part A: Polym. Chem. 1996, 34, 1589.

(16) Prucker, O; Ruhe, J. Macromolecules 1998, 31, 592

(17) Prucker, O.; Ruhe, J. Macromolecules 1998, 31, 602

(18) Zhang, H.; Ruhe, J. Macromolecules 2003, 36, 6593.

(19) Ruhe, J.; Ballauff, M.; Biesalski, M.; Dziezok, P.; Grohn, F.; Johannsmann, D.; Houbenov, N.; Hugenberg, N.; Konradi, R.; Minko, S.; Motornov, M.; Netz R.; Schmidt, M.; Seidel, C.; Stamm, M.; Stephan, T.; Usov, D.; Zhang, H. Adv. Polym. Sci. 2004, 165, 79.

(20) Kurihara, K.; Kunitake, T.; Higashi, N.; Niwa, M. Langmuir 1992, 8, 2087.

(21) Prinz, C.; Muller, P.; Maaloum, M. Macromolecules 2000, 33, 4896.

(22) Ahrens, H.; Forster, S.; Helm, C. Phys. Rev. Lett. 1998, 81, 4172

(23) Levicky, R. Ph.D. Thesis, University of Minnesota, 1996. 


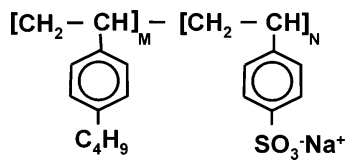

Figure 1. Schematic of the structure of PtBS-NaPSS.

while the polyelectrolyte block extends away. Depending on the tethering density, the polyelectrolyte chains exhibit two limiting conformations. At low adsorption densities, an "isolated coil" structure is formed, where neighboring chains do not overlap. At high grafting densities, the chains overlap, driving an additional stretching normal to the surface and forming a "polymer brush". ${ }^{24}$ Our previous studies showed that the ionic strength is a critical factor influencing the adsorption behavior of hydrophilichydrophobic block copolymers. ${ }^{8-10}$ We found that the addition of salt $(\mathrm{NaCl})$ to the polyelectrolyte solution screens the interchain repulsion, and a certain amount of salt has to be added before any measurable adsorption occurs. When the concentration of $\mathrm{NaCl}$ reaches about $0.15 \mathrm{M}$, significant adsorption takes place. In addition to the ionic strength, the nature of the surface/interface also significantly affects the adsorption behavior and the structure of adsorbed polyelectrolyte layers.

In this paper, we report the results of force measurements between adsorbed layers of hydrophobic/hydrophilic diblock copolymers. The systems studied are diblock copolymers of poly( $t$-butylstyrene) and poly(styrene sulfonate) (PtBS-PSS) (the chemical structure is shown Figure 1) adsorbed on both a hydrophilic surface (bare mica) and a hydrophobic surface $(\mathrm{N}$ octadecyltriethoxysilane (OTE)-modified mica). The paper is organized as follows. We first describe the method used to hydrophobize the mica surface with OTE, and then we present and compare the force curves of polyelectrolyte layers adsorbed onto bare mica and OTE-modified mica surfaces, respectively, obtained by using the surface force apparatus (SFA). We examine and discuss factors which determine the structure and force profiles of adsorbed polyelectrolyte layers: salt concentration, molecular weight of polyelectrolytes, and substrate properties. Finally, we compare our experimental results to theoretical predictions, which allow us to propose different structures of the adsorbed layer when adsorption takes place onto bare mica or OTE.

\section{Experimental Section}

Materials. Mica was obtained from S and J Trading, and was cleaved into $1 \mathrm{~cm}^{2}$ step-free square coupons with thickness of 1-3 $\mu \mathrm{m}$, which is required for the force measurement experiments. OTE was purchased from United Chemical Technologies, Inc. Aqueous solutions used for self-assembly were prepared from pure water obtained by distillation, deionization, and reverse osmosis (Milli-Q water purification system). The resulting resistance of the water was $18.2 \mathrm{M} \Omega / \mathrm{cm}$. Sodium chloride or sodium nitrate salt (Aldrich, $>99.99 \%$ pure) was then added to the polyelectrolyte aqueous solution to a concentration of $0.3 \mathrm{M}$. This high concentration of added salt was used to screen electrostatic interactions during adsorption and, thus, to maximize the adsorbed amount.

The diblock copolymers used in this study was PtBS-NaPSS of various molecular weights and compositions. The PtBS block is strongly hydrophobic and insoluble in aqueous media, while the NaPSS block is hydrophilic and soluble in water. The diblock copolymers were made highly asymmetric (small PtBS block and very large NaPSS block) to ensure water solubility. They have polydispersity ratios $<1.05$ based upon size exclusion chromatography and matrix-assisted laser desorption/ionization time-of-flight mass spectrometry data, with sulfonation levels in the NaPSS blocks of around $90 \%$ based upon elemental analysis (Table 1). The

(24) Milner, S. T. Science 1991, 251, 905. copolymers were dissolved in salty water to a concentration of 80 $\mathrm{ppm}$, and then the solution was stirred for at least 4 days at $60{ }^{\circ} \mathrm{C}$ to ensure that any nonmicellar aggregates were dissolved. ${ }^{25}$

Hydrophobic Surface Preparation. Langmuir isotherms and LB deposition were carried out using a KSV 5000 Langmuir trough. A LB monolayer of OTE was deposited onto the surface of $1 \mathrm{~cm}^{2}$ mica sheets, which were glued to cylindrically curved glass lenses beforehand. Before the LB deposition, the mica surface was treated with argon/water-vapor plasma for $30 \mathrm{~min}$ at $20 \mathrm{~W}$ to generate $\mathrm{SiOH}$ groups on the surfaces. The water-vapor flow rate was such that the pressure in the plasma chamber (initially at $34 \mathrm{mTorr}$ ) was maintained at 54 mTorr.

The protocol for producing the self-assembled monolayers of OTE developed by Wood and Sharma ${ }^{26}$ was adopted but somewhat modified for the deposition. OTE was first dissolved in chloroform/ methanol (95:5 in volume) at a concentration of $2 \mathrm{mg} / \mathrm{mL}$. A $35 \mu \mathrm{L}$ solution was spread onto a subphase of neutral $\mathrm{pH} 7$ and allowed to stand for $30 \mathrm{~min}$ before compressing the film to $30 \mathrm{mN} / \mathrm{m}$ at a rate of $10 \mathrm{~mm} / \mathrm{s}$. The $\mathrm{pH}$ of the subphase was then lowered to $\mathrm{pH}$ $2 \pm 0.5$ using sulfuric acid after the film was compressed at $30 \mathrm{mN} / \mathrm{m}$ and the compression was maintained for $90 \mathrm{~min}$. Monolayers of OTE were sequentially deposited onto the mica surface at a velocity of $1 \mathrm{~mm} / \mathrm{min}$ and pressure of $30 \mathrm{mN} / \mathrm{m}$ with a transfer ratio of 0.95 \pm 0.05 . After the deposition, the surfaces were dried in a clean air stream for $20 \mathrm{~min}$ and then were moved into an oven and annealed at $45^{\circ} \mathrm{C}$ for $30 \mathrm{~min}$, then at $70{ }^{\circ} \mathrm{C}$ for $30 \mathrm{~min}$, and finally at 100 ${ }^{\circ} \mathrm{C}$ for $90 \mathrm{~min}$. This protocol, as we shall see later, produces a relatively smooth and homogeneous OTE monolayer on the mica surface. The resulting contact angle of the hydrophobic OTE monolayer was about $110^{\circ}$, in accord with other studies. ${ }^{26,} 27$

AFM Imaging. Images of OTE surfaces were obtained under water using a Dimensional 3100 AFM with a liquid cell setup provided by Digital Instruments.

Normal Force Measurements. Force measurements were carried out on a surface forces apparatus, which is similar to the design of Israelachvili. ${ }^{28,29}$ This apparatus permits accurate measurement of interaction forces, $F(D)$, as a function of the surface separation, $D$. The basic techniques and the detailed experimental procedures involved in force measurements have been described elsewhere. ${ }^{30,31}$ At the beginning of each experiment, the mica surfaces were brought into contact in air. Observation of flat adhesive contact ensured that surfaces were free of contamination. The thickness of the smooth mica sheets was measured from the positions of the interference fringes. Deposition of OTE was carried out as described above, and observation of flat adhesive contact between the two OTE monolayers was observed. The measured thickness of the OTE monolayer (20 $\pm 5 \AA$ ) was in good accord with the literature. ${ }^{26,27}$ The polyelectrolyte aqueous solution was filtered with a $0.45 \mu \mathrm{m}$ cellulose filter and added into the SFA chamber and the substrates (bare mica surface or OTE-modified mica surface) were then immersed. Since water is a poor solvent for the PtBS block and the added salt screens the electrostatic interaction between polyelectrolyte segments, the PtBS block will preferentially adsorb on the surface of the substrate and the NaPSS blocks extend into the aqueous media. After $14 \mathrm{~h}$, the bulk polyelectrolyte solution was replaced with pure water to stop further adsorption. $\mathrm{NaCl}$ (or $\mathrm{NaNO}_{3}$ ) solution was added to the SFA chamber until the concentration reached about $0.3 \mathrm{M}$. The forcedistance profile ( $F / \mathrm{R}$ vs $D$ ) was obtained at this concentration, where $R$ is the mean curvature of the cross-cylindrical lenses. The bulk salt concentration was gradually diluted, and force-distance profiles at 7339.

(26) Wood, J.; Sharma, R. Langmuir 1994, 10, 2307.

(27) Peanasky, J.; Schneider, H. M.; Granick, S.; Kessel, C. R. Langmuir $1995,11,953$.

(28) Israelachvili, J. N.; Adams, G. E. J. Chem. Soc., Faraday Trans. 1 1978, 74,975 .

(29) Israelachvili, J. N. J. Colloid Interface Sci. 1973, 44, 259.

(30) Hadziioannou, G.; Patel, S.; Granick, S.; Tirrell, M. J. Am. Chem. Soc. 1986, 108, 2869.

(31) Balastre, M.; Li, F.; Schorr, P.; Yang, J.; Mays, J. W.; Tirrell, M. Macromolecules 2002, 35, 9480. 
Table 1. Molecular Information of PtBS-NaPSS Diblock Copolymers and Tethering Density Obtained by the SFA Dry-Layer Measurement (a) and by Ellipsometry (b) ${ }^{a}$

\begin{tabular}{|c|c|c|c|c|c|}
\hline polymer name & MT1 & MT2 & MT3 & MT5 & MT6 \\
\hline degree of polymerization of PtBS block & 54 & 26 & 27 & 15 & 15 \\
\hline degree of polymerization of NaPSS block, $N$ & 879 & 403 & 747 & 438 & 612 \\
\hline degree of sulfonation, $\beta$ & $93 \%$ & $89 \%$ & $87 \%$ & $85 \%$ & $84 \%$ \\
\hline polydispersity & 1.03 & 1.03 & 1.04 & 1.03 & 1.04 \\
\hline tethering density (bare mica surface), $\sigma\left(10^{15} \text { chains } / \mathrm{m}^{2}\right)^{a}$ & $2.3 \pm 0.5$ & $5.8 \pm 1.2$ & $2.8 \pm 0.6$ & $6.3 \pm 1.3$ & - \\
\hline tethering density (OTE-modified mica), $\sigma\left(10^{15} \text { chains } / \mathrm{m}^{2}\right)^{a}$ & - & - & $10.1 \pm 2$ & $15.4 \pm 3.1$ & $12.6 \pm 2.5$ \\
\hline tethering density (OTE-modified mica), $\sigma\left(10^{15} \text { chains } / \mathrm{m}^{2}\right)^{b}$ & $6.9 \pm 0.2$ & - & $7.4 \pm 0.2$ & $12.5 \pm 0.4$ & $9.5 \pm 00.3$ \\
\hline
\end{tabular}

${ }^{a}$ The PtBS-NaPSS diblock copolymer was adsorbed from aqueous solution with $0.3 \mathrm{M}$ added $\mathrm{NaNO}_{3}$ or $\mathrm{NaCl}$ to two different substrates. The adsorption time was $14 \mathrm{~h}$.

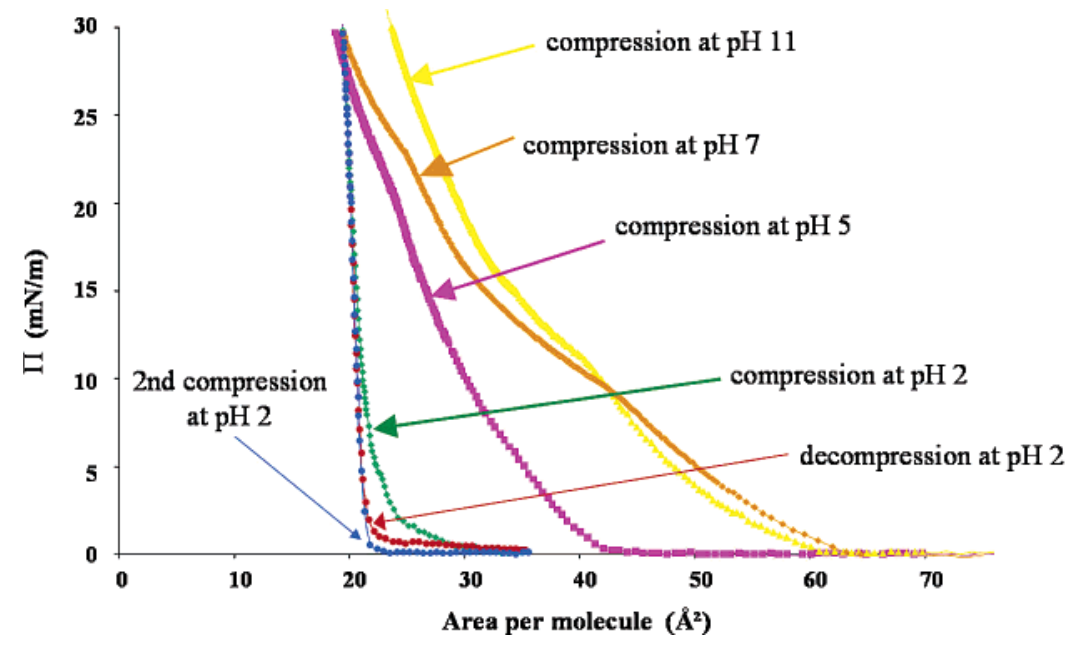

Figure 2. Surface pressure (П) versus area per molecule isotherms for monolayers of OTE on the aqueous subphases of different pH at $25 \pm 0.2^{\circ} \mathrm{C}$.
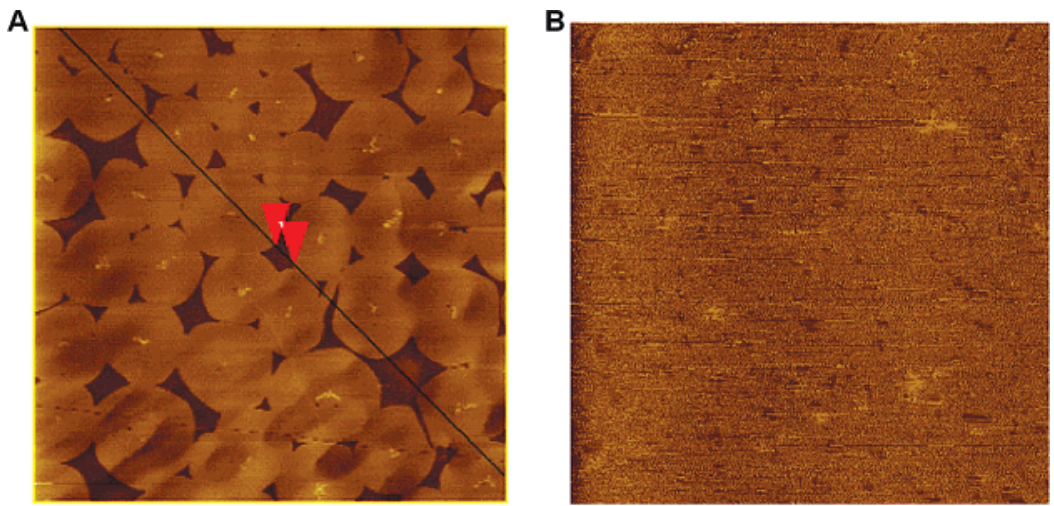

Figure 3. Tapping mode AFM image $(30 \mu \mathrm{m} \times 30 \mu \mathrm{m})$ of OTE monolayer deposited on the mica surface following the Wood and Sharma protocol (A) and following our protocol (B).

other salt concentrations were measured. The precise salt concentration in the bulk solution was measured using a conductivity probe. The temperature inside the SFA was controlled at $30 \pm 0.2^{\circ} \mathrm{C}$ for all experiments. All sets of SFA data in this paper represent several compression/separation cycles performed on the same spot, as well as data from different areas of different assembled layers.

\section{Results and Discussion}

Langmuir Isotherms of OTE. Figure 2 shows Langmuir isotherms of OTE layers with subphase of different $\mathrm{pHs}(\mathrm{pH} 2$, $5,7,11)$. The isotherm at $\mathrm{pH} 2$ has been reported elsewhere ${ }^{26}$

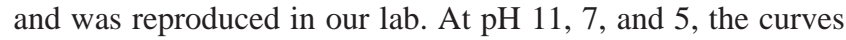
exhibit a liquid expanded (LE) phase, followed by a more compressible phase (at about $42 \AA^{2}$ ), a liquid condensed phase (LC) (at about $26 \AA^{2}$ ), and a collapse (at about $20 \AA^{2}$ ) corresponding to a molecular area equivalent to the cross-sectional area of a single hydrocarbon tail. ${ }^{32}$ At lower $\mathrm{pH}$, OTE hydrolyzes and polymerizes laterally in the plane of the interface, leading to predominantly condensed phases. The expanded region becomes larger when the $\mathrm{pH}$ is increased, suggesting that less and less polymerization occurs.

AFM Images of OTE Monolayers. Figure 3A shows a tapping mode image of an annealed OTE monolayer deposited on the mica surface, reproduced in our lab following a published protocol. ${ }^{26,33}$ In this case, OTE molecules were directly spread onto a subphase of $\mathrm{pH} \mathrm{2,} \mathrm{compressed,} \mathrm{and} \mathrm{then} \mathrm{deposited} \mathrm{onto}$ the mica surface that had been treated with argon/water-vapor plasma. The image shows polymerized OTE islands with diameter of about $6 \mu \mathrm{m}$ in contact, with unpolymerized OTE in between. This resulting morphology is not really understood and could be

(32) Evans, D. F.; Wennerström, H. The Colloidal Domain: Where Physics, Chemistry, Biology, and Technology Meet; Wiley-VCH: Weinheim, 1999.

(33) Schneider, J.; Dori, Y.; Tirrell, M.; Sharma, R. Thin Solid Films 1998, $329,772$. 
explained by an uncontrolled nucleation-growth mechanism, which takes place before compressing the OTE molecules. ${ }^{33,} 34$

We have developed a modified procedure that avoids island formation. The early growth can be blocked by spreading the OTE molecules onto a subphase of $\mathrm{pH}$ 7-8 (in order to prevent hydrolysis and condensation) followed by a slow compressing of the molecules to force them to be ordered and in contact. After this process, we initiated the hydrolysis/condensation reactions by dropping the $\mathrm{pH}$ of the subphase to $\mathrm{pH} 2$ while the OTE molecules were still densely packed. Ninety minutes was allowed for hydrolysis/condensation reactions before the OTE monolayer was transferred to the mica surface. Figure 3B is a tapping mode AFM image of the annealed OTE layer treated by using this modified procedure. It clearly shows that the surface is made of a more uniform OTE layer with smaller defect regions, suggesting that this protocol favors a uniform growth rather than an island growth mechanism. Achieving this structure requires a compact arrangement of the OTE molecules before any hydrolysis and condensation reactions take place.

Adsorption. The concentrations of PtBS-NaPSS solutions used in all our experiments were about $80 \mathrm{ppm}$, which is above the cmc of these diblock copolymers, ${ }^{35-37}$ so micelles will be present in solution. Recent studies in our group indicate that the micelles do not directly adsorb to hydrophilic substrates such as bare mica surfaces. ${ }^{38}$ Instead, the free chains in solution adsorb, which drives a breakup of the micelles to maintain a constant concentration of free chains in the solution. For OTE-modified mica surfaces, the adsorption mechanism was shown to be different since the hydrophobic micelle core can overcome the barrier energy induced by its charged shell, directly adsorb, and rearrange over the surface. ${ }^{38,39}$ The adsorption kinetics and final adsorbed amounts obtainable with these block copolymers from aqueous solution have been studied in our lab by ellipsometry. ${ }^{8-10,38,39}$

The adsorbed amount can also be measured by the SFA by measuring the dry layer thickness of the adsorbed polyelectrolyte layers (Table 1). When all the force measurements on a particular sample have been finished, the solvent is drained from the chamber and filtered nitrogen passes through the apparatus overnight to dry the adsorbed polymer layers. The surfaces are then brought into contact and the thickness of the dry polymer layer was measured. At very similar adsorption conditions, the adsorbed amount measured by the SFA is approximately $20-40 \%$ higher than that by measured by ellipsometry, as shown in Table 1, because the polyelectrolyte layers are extremely hydrated and some residual water will always remain in the "dry" layers at ambient temperature and pressure. Thus, the values obtained by the SFA are less accurate than the ellipsometric measurements. However, ellipsometric measurements of adsorption on bare mica surfaces are challenging, owing to birefringence of mica, and therefore, in this case, only values found by the SFA are reported here.

The adsorbed amount depends strongly on the substrate affinity. Because the bare mica surface is hydrophilic and weakly negatively charged in aqueous media, adsorption onto this surface is only slightly more favorable for the PtBS blocks than remaining in aqueous solution. This leads to relatively low adsorbed amounts

(34) Wood, J.; Sharma, R. Langmuir 1995, 11, 4797

(35) Guenoun, P.; Lipsky, S.; Mays, J. W.; Tirrell, M. Langmuir 1996, 12, 1425.

(36) Guenoun, P.; Davis, H. T.; Tirrell, M.; Mays, J. W. Macromolecules 1996, 29, 3965.

(37) Guenoun, P.; Delsanti, M.; Gazeau, D.; Mays, J. W.; Cook, D. C.; Tirrell, M.; Auvray, L. Eur. Phys. J. B 1998, 1, 77.

(38) Toomey, R. Ph.D. Thesis, University of Minnesota, 2002.

(39) Toomey, R.; Mays, J.; Holley, D. W.; Tirrell, M. Macromolecules 2005. $38,5137$. of the polymer chains. When the adsorption takes place onto hydrophobically modified mica substrates, the affinity between the hydrophobic adsorbing block and the hydrophobic OTE surface increases, hence the adsorbed amount of polymer chains increases by a factor of 2-3. Structure and force profiles of the polyelectrolyte layers adsorbed on these two different surfaces will be discussed later. In the reminder of this paper, we will use the term "tethered" to refer to the diblock copolymers connected to the substrate surface through the adsorption of the anchoring PtBS blocks.

Force Profiles. The normal forces between the polyelectrolyte with various molecular weights, adsorbed onto the bare mica surface and OTE-modified mica surface, respectively, were measured with the SFA at different added salt concentrations. Only the force curves of polyelectrolyte layers adsorbed on the bare mica surface is reported here, while those force curves of polyelectrolytes adsorbed onto OTE-modified mica surface can be found in our previous paper. ${ }^{31}$

For polyelectrolytes tethered to the OTE-modified mica surface, our studies show that the adsorbed layers are within the brush regime and can be well described by the scaling brush theory. ${ }^{31}$ The measured forces between brush layers can be regarded as the contributions of steric interactions and short-ranged electrostatic interactions, while long-ranged electrostatic double-layer forces have not been detected. A large fraction of counterions condense to the polyelectrolyte backbones, while the rest of the counterions are trapped inside the brush. We successfully reduced all our data into a "universal brush height curve", which considers all factors that affect the brush height. The force data of polyelectrolytes tethered to a bare mica surface, however, exhibit features different from the brush behavior, as will be described below.

When the forces between the two opposing polyelectrolyte layers are measured, a hysteresis is sometimes observed between different compression/separation cycles for the same polymer at the same added salt concentration. This hysteresis effect has been observed previously in our group in studies of polyelectrolyte brushes on mica using the SFA ${ }^{40,41}$ but not in layers adsorbed on OTE. ${ }^{31}$ Figure 4 shows a typical hysteresis between the first compression and all subsequent compressions made on a given spot of the sample. The force curves are shown to shift to smaller separation distance upon the first compression at a given spot and then become reproducible for all subsequent compression/ separation cycles. The magnitude of this effect is relatively small, reversible, and could relax with time (time scale of hours).

Figure 5 shows the reproducible forces versus distance profiles measured at different salt concentrations between the two layers of PtBS-NaPSS diblock copolymer of different molecular weights on the bare mica surface adsorbed from aqueous polymer solution with $0.3 \mathrm{M} \mathrm{NaCl}$ (or $\mathrm{NaNO}_{3}$ ). The forces are purely repulsive for both compression and separation. The onset of forces occurs at a distance well below the contour lengths of the chains and slightly larger than twice the radius of gyration of NaPSS in solution at similar added salt concentrations (Table 2). As the molecular weight of the NaPSS chains is increased at a given salt concentration, the range of the normal forces increases. An increase of the range of the force profile is observed upon decreasing the salt concentration due to a decrease of the screening of the electrostatic repulsion. It is interesting to note that, for the same salt concentration and molecular weight, the onset of the interaction forces, $D_{0}$, of polyelectrolytes tethered to the OTE-

(40) Watanabe, H.; Patel, S. S.; Argillier, J. F.; Parsonage, E. E.; Mays, J.; Dan-Brandon, N.; Tirrell, M. Mater. Res. Soc. Symp. Proc. 1992, 249, 255.

(41) Mao, G. Ph.D. Thesis, University of Minnesota, 1994. 


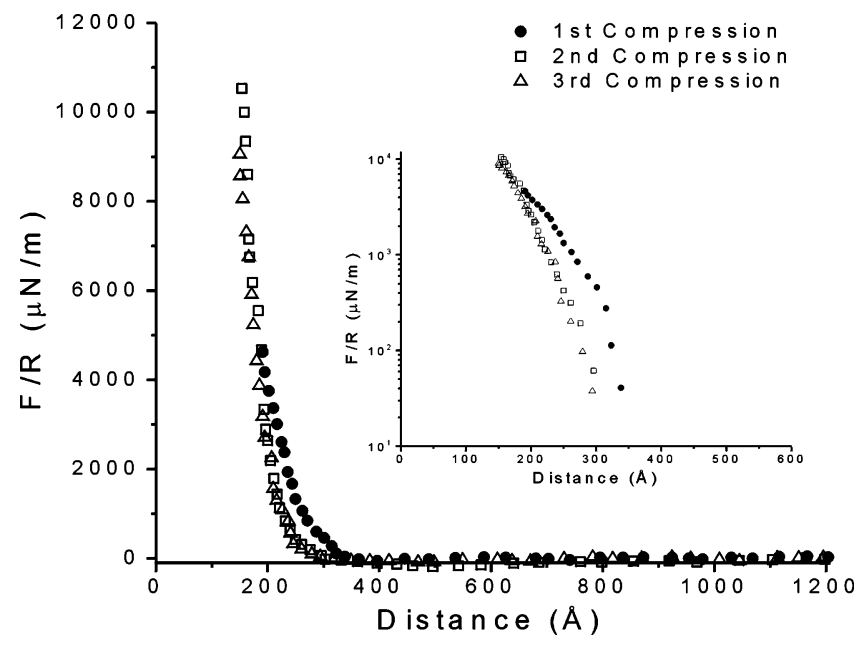

Figure 4. Normal forces between PtBS-NaPSS (MT2) layers tethered to bare mica surfaces and immersed in $0.3 \mathrm{M} \mathrm{NaCl}$ aqueous media. The hysteresis observed here between the first and subsequent compressions would relax with time $(2-3 \mathrm{~h})$ so that the original compression curve was again obtained. The inset is a semi-log plot of the same curves.

modified mica surface occurs at larger distance than to the bare mica surface. This difference might be explained in terms of the structure of the polyelectrolyte layers, reflecting the differences in the tethering density.

An important question to be addressed is whether the forces measured between the two opposing polyelectrolyte layers consist of a component of the electrical double forces that extends beyond the confinement of the polymer segment distribution. In other words, does the onset of the normal forces, $D_{0}$, equal twice the equilibrium height of the adsorbed layer, $2 L_{0}$ ? For polyelectrolyte brushes adsorbed on the OTE-modified mica surface, we demonstrated in the previous paper ${ }^{31}$ that no electrical doublelayer forces were detected and all the counterions were trapped inside the brushes, which is in good agreement with the smallangle $\mathrm{X}$-ray scattering experiment ${ }^{42}$ and the molecular dynamics simulations. ${ }^{43}$ Following the argument proposed by Zhulina et al. ${ }^{44}$ we can analyze the position of the counterion cloud for polyelectrolytes adsorbed to the bare mica surface. The onset of the measured interaction forces, which was determined from the experimental force curves, can also be regarded as the distance where the counterion clouds of the two opposing polyelectrolyte layers start to overlap (if assuming the counterion cloud extends beyond the adsorbed layer). Hence, the counterions of a single polyelectrolyte layer are distributed in the space between the mica surface and $D_{0} / 2$. Another important length scale for this system is the Gouy-Chapmann length,

$$
\Lambda=\frac{1}{2 \pi l_{B} \sigma \alpha N}
$$

where $l_{\mathrm{B}}$ is the Bjerrum length $(7.14 \AA$ for water $),{ }^{45} \sigma$ is the tethering density in chains per unit area which can be calculated from the measured adsorbed amount, and $N$ is the degree of polymerization of the PSS block. Here $\alpha$ is the fraction of free counterions (ratio of free counterions concentration to the concentration of polymer segments) and is modified for counterion condensation. ${ }^{46}$ Since polyelectrolytes adsorbed to the bare mica

(42) Bendejacq, D.; Ponsinet, V.; Joanicot, M. Eur. Phys. J. E 2004, 13, 3 (43) Seidel, C. Macromolecules 2003, 36, 2536.

(44) Zhulina, E. B.; Borisov, O. V. J. Chem. Phys. 1997, 107, 5952.

(45) Israelachvili, J. N. Intermolecular And Surface Forces; Academic Press: London, 1992 surface are not as densely adsorbed as for the brush structure, it is reasonable to assume the Manning condensation theory is still valid for this configuration, which gives

$$
\alpha=\frac{2.5}{\beta l_{B}}=\frac{0.35}{\beta}
$$

where $\beta$ is the degree of sulfonation of the polyelectrolyte chains. Therefore, the Gouy-Chapmann length, $\Lambda$, which characterizes the thickness of the counterion cloud above the charged planar surface, was calculated for each material, and the results are given in Table 2. Clearly, $D_{0} / 2 \gg \Lambda$, which means the Coulomb attraction retains the counterions inside the adsorbed layer; hence, $D_{0} / 2$ can indeed be interpreted as $L_{0}$, where $L_{0}$ refers to the equilibrium height of the adsorbed polyelectrolyte layer at a given salt concentration. For each salt concentration, a height of the polyelectrolyte layer, $L_{0}$, was obtained from the onset of the interaction forces and is reported in Table 2 .

Degree of Overlap. The degree of overlap between chains, $\sigma^{*}$, can be quantified as $\sigma^{*}=L_{0}^{2} / \mathrm{A}$, where $A$ is the average area per chain; hence, $A=1 / \sigma$. When $\sigma^{*} \ll 1$, the polyelectrolytes are sparsely tethered and adopt a random orientation normal to the surface (isotropically stretched regime). ${ }^{47}$ When $\sigma^{*} \approx 1$, the chains take on a strong orientation normal to the tethering surface due to intermolecular interactions but are only stretched in the normal direction by intramolecular interactions. The height of the layer at this point is thought to still be approximately the radius of gyration, $R_{\mathrm{g}}$, of the free chains in solution (ordered sticks). ${ }^{47}$ Finally, when $\sigma^{*} \gg 1$, the intermolecular interactions between chains promote an additional stretching normal to the surface and the layer follows the physics of polyelectrolyte brushes.

For polyelectrolytes adsorbed on the bare mica surface, the values of $\sigma^{*}$ range from 3 at high salt concentrations to 25 at low salt concentrations (Table 2). This indicates that the selfassembly of PtBS-NaPSS diblock copolymer on bare mica surfaces from an aqueous polyelectrolyte solution with $0.3 \mathrm{M}$ $\mathrm{NaCl}$ does not appear to produce a very dense polyelectrolyte brush. Though 25 may appear at first thought to be a high value of $\sigma^{*}$, it is low compared to the values attained on hydrophobic surfaces (see below). Instead, some intermediate structure is formed with the height of the layer scaling with the radius of gyration of the NaPSS block. Furthermore, the almost constant value of $L_{0} / R_{\mathrm{g}}$ with decreasing salt concentration suggests that the chains hardly enter into the brush regime even though the range of the electrostatic interaction is increasing.

When adsorption takes place onto the OTE-modified mica surface, the degree of overlap, $\sigma^{*}$, is much greater and varies between 20 at $0.3 \mathrm{M} \mathrm{NaCl}$ (or $\mathrm{NaNO}_{3}$ ) up to 200 at very low salt concentration (Table 3 ). Values of $L_{0} / R_{\mathrm{g}}$ also significantly vary as the salt concentration decreases, implying greater stretching of the chains than in the previous case with decreasing the salt concentration, suggesting an additional stretching of the chains probably due to an higher tethering density.

Figure 6 shows the degree of stretching of the chains, defined as $L_{0} / R_{\mathrm{g}}$, as a function of the degree of overlap, $\sigma^{*}$, for adsorption to both the bare mica and the OTE-modified mica surfaces. In this $\log -\log$ plot, all the points corresponding to adsorption to the OTE-modified mica surface fall on a straight line with a slope of about 0.2 , while most points from adsorption to the bare mica surface form a plateau at lower $L_{0} / R_{\mathrm{g}}$ values.

(46) Manning, G. S. J. Chem. Phys. 1969, 51, 924.

(47) Borisov, O. V.; Zhulina, E. B.; Birshtein, T. M. Macromolecules 1994, $27,4795$. 

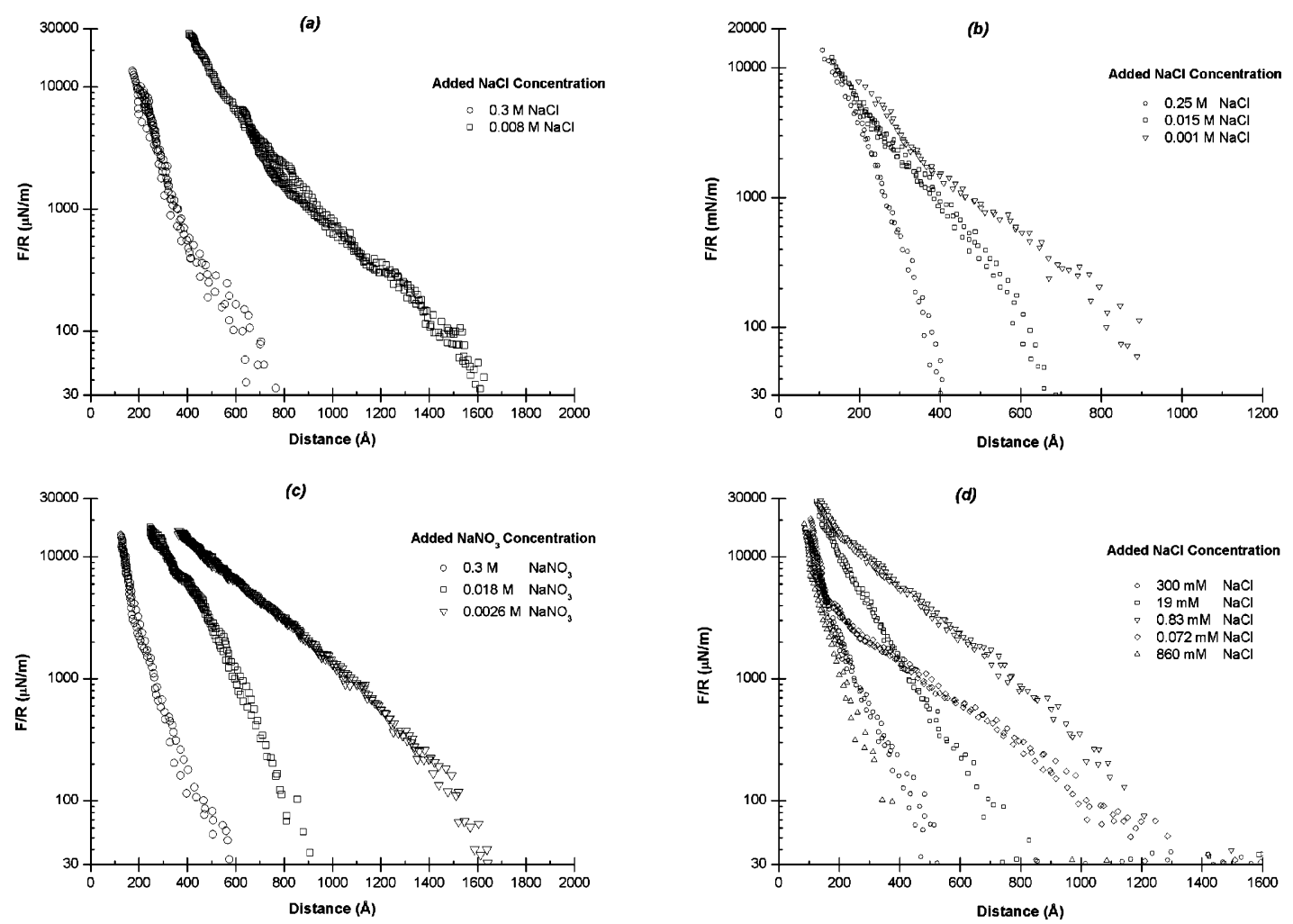

Figure 5. Normal forces between four PtBS-NaPSS copolymer layers tethered to bare mica surfaces at different added salt concentrations: (a) MT1, (b) MT2, (c) MT3, and (d) MT5. At each salt concentration, forces were measured for both compression and separation.

Table 2. Characterization of PtBS-NaPSS Layers Tethered on Bare Mica Surfaces at Different Added Salt Concentrations, $C_{s}$

\begin{tabular}{cccccccc}
\hline \multirow{2}{*}{ polymer } & $N$ & $\begin{array}{c}\Lambda \\
(\AA)\end{array}$ & $\begin{array}{c}\text { added salt } \\
\text { concn, } C_{s}(\mathrm{M})\end{array}$ & $\begin{array}{c}R_{g} \\
(\AA)^{a}\end{array}$ & $\begin{array}{c}L_{0} \\
(\AA)\end{array}$ & $\sigma^{*}$ & $L_{0} / R_{\mathrm{g}}$ \\
\hline MT1 & 879 & 2.9 & $3.0 \times 10^{-1}$ & 309 & 425 & $4.1 \pm 0.8$ & $1.4 \pm 0.07$ \\
& & & $8.0 \times 10^{-3}$ & 599 & 838 & $16 \pm 3.2$ & $1.4 \pm 0.07$ \\
MT2 & 403 & 2.4 & $2.5 \times 10^{-1}$ & 188 & 225 & $3 \pm 0.6$ & $1.2 \pm 0.06$ \\
& & & $1.5 \times 10^{-2}$ & 296 & 375 & $8.2 \pm 1.6$ & $1.3 \pm 0.07$ \\
& & & $1.0 \times 10^{-3}$ & 318 & 550 & $17.6 \pm 3.5$ & $1.7 \pm 0.09$ \\
MT3 & \multirow{2}{*}{247} & 2.6 & $3.0 \times 10^{-1}$ & 279 & 375 & $3.9 \pm 0.8$ & $1.3 \pm 0.07$ \\
& & & $1.8 \times 10^{-2}$ & 509 & 575 & $9.3 \pm 1.9$ & $1.1 \pm 0.06$ \\
& & & $2.6 \times 10^{-3}$ & 557 & 875 & $21.5 \pm 4.3$ & $1.6 \pm 0.08$ \\
MT5 & \multirow{2}{*}{438} & 2.0 & $8.6 \times 10^{-1}$ & 170 & 225 & $3.2 \pm 0.6$ & $1.3 \pm 0.07$ \\
& & & $3.0 \times 10^{-1}$ & 191 & 263 & $4.3 \pm 0.9$ & $1.4 \pm 0.07$ \\
& & & $1.9 \times 10^{-2}$ & 322 & 425 & $11.4 \pm 2.3$ & $1.3 \pm 0.07$ \\
& & & $8.3 \times 10^{-4}$ & 349 & 625 & $24.6 \pm 4.9$ & $1.8 \pm 0.09$ \\
& & & $7.2 \times 10^{-5}$ & 349 & 625 & $24.6 \pm 4.9$ & $1.8 \pm 0.09$
\end{tabular}

${ }^{a}$ The radius of gyration of free NaPSS chains, $R_{g}$, was calculated on the basis of the studies in refs 48 and 51 by considering that both the counterions and the added salt contribute the ionic strength.

This graph shows that the polyelectrolyte chains start to feel an additional stretching when the degree of overlap, $\sigma^{*}$, is larger than 10 . The power law relationship, $L_{0} / R_{\mathrm{g}} \propto \sigma^{* 0.2} \propto\left(L_{0}{ }^{2} / A\right)^{0.2}$ $\propto\left(L_{0}^{2} \sigma\right)^{0.2}$, which can be translated to $L_{0} \propto \sigma^{1 / 3}$, agrees well with the brush theory, indicating that a brush structure is indeed formed through adsorbing polyelectrolytes to the OTE-modified mica surface. For points under $\sigma^{*}=10$, the degree of stretching shows little dependence on $\sigma^{*}$, corresponding to adsorption to the bare mica surface. Therefore, we can conclude that, in this case, the tethering density is not high enough to drive an additional stretching of the chains and the polymer layer is made of sparsely tethered chains. The increase of the layer height with decreasing salt concentration tends to mirror the predicted increase in the radius of gyration of the NaPSS chains with decreasing salt concentration. This suggests that intramolecular interactions are dominating over intermolecular interactions when adsorption takes place onto the bare mica surface.
Comparison with Scaling Models. For sparsely tethered polyelectrolyte chains, it is reasonable to assume the counterion condensation obeys the Manning theory, which leads to $\alpha=$ $0.35 / \beta$, and the values of $\alpha$ are very close to 0.40 for all the diblock copolymers of different molecular weight and degree of sulfonation in this study. For the brush structures, the values of $\alpha$, calculated from the experimental data, ${ }^{31}$ are also very close to 0.18 for different samples studied. Therefore, for each case, it is reasonable to assume $\alpha$ is constant for all adsorbed diblock copolymers studied. Figure 7 shows the plot of $L_{0}$ as a function of $N$ at very low added salt concentrations. In this low-salt limit, the scaling theory predicts $L_{0}$ scales $N$ to the first power $(v=$ 1) for a polyelectrolyte brush, as well as for strongly charged, sparsely tethered chains. The slope appears to be $v=1$ when adsorption takes place onto the OTE-modified mica surface, in good accord with the first power dependence expected by the scaling brush theory; whereas $v=0.7-1$ when adsorption takes place onto the hydrophilic mica surface, lower than expected for the sparse tethering polyelectrolyte chains. The relationship between $R_{\mathrm{g}}$ and $N$ has been determined experimentally for polyelectrolytes in solutions such as $\mathrm{NaPSS}^{48}$ and through simulations for weak polyacids such as PAA.49,50 Those relationships allows one to determine how "rodlike" the charged chains are behaving $\left(R_{\mathrm{g}} \propto N\right)$ and the effective "good" $\left(R_{\mathrm{g}} \propto\right.$ $\left.N^{0.6}\right)$ and "theta" $\left(R_{\mathrm{g}} \propto N^{0.5}\right)$ ionic strength conditions. The data suggest that the NaPSS chains adopt a configuration similar to a neutral chain in a theta solvent at $3.1 \mathrm{M} \mathrm{NaCl}$ and similar to a neutral chain in a good solvent at $0.15 \mathrm{M} \mathrm{NaCl}$. As the ionic strength is reduced further to $2 \mathrm{mM}$, the NaPSS chains start to exhibit significant electrostatic stretching but remain well below the rodlike limit. Our results are in good agreement with the dilute solution measurements of Wang et al. on NaPSS. ${ }^{48}$

(48) Wang, L.; Yu, H. Macromolecules 1988, 21, 3498.

(49) Winkler, R. G.; Gold, M.; Reineker, P. Phys. Rev. Lett. 1998, 80, 3731

(50) Schafer, H.; Seidel, C. Macromolecules 1997, 30, 6658.

(51) Benoit, H.; Doty, P. J. Phys. Chem. 1954, 57, 958. 
Table 3. Characterization of PtBS-NaPSS Layers Tethered on the OTE-Modified Mica Surface at Different Added Salt Concentrations, $C_{s}$.

\begin{tabular}{|c|c|c|c|c|c|c|c|c|c|c|c|}
\hline polymer & $N$ & $\begin{array}{c}\text { added salt } \\
\text { concn, } C_{s}(\mathrm{M}) \\
\end{array}$ & $\begin{array}{r}R_{g} \\
(\AA)^{a} \\
\end{array}$ & $\begin{array}{l}L_{0} \\
(\AA)^{b} \\
\end{array}$ & $\sigma^{*}$ & $L_{0} / R_{\mathrm{g}}$ & $\begin{array}{c}\text { added salt } \\
\text { concn, } C_{s}(\mathrm{M})\end{array}$ & $\begin{array}{r}R_{g} \\
(\AA)^{a} \\
\end{array}$ & $\begin{array}{c}L_{0} \\
\left(\AA{ }^{b}\right.\end{array}$ & $\sigma^{*}$ & $L_{0} / R_{\mathrm{g}}$ \\
\hline \multirow[t]{3}{*}{ MT1 } & 879 & $3.2 \times 10^{-1}$ & 304 & 615 & $26.1 \pm 1.3$ & $2.0 \pm 0.1$ & $2.7 \times 10^{-2}$ & 511 & 1410 & $137.1 \pm 6.9$ & $2.8 \pm 0.14$ \\
\hline & & $1.4 \times 10^{-1}$ & 376 & 785 & $42.5 \pm 2.1$ & $2.1 \pm 0.11$ & $9.1 \times 10^{-3}$ & 581 & 1690 & $197 \pm 9.9$ & $2.9 \pm 0.15$ \\
\hline & & $5.3 \times 10^{-2}$ & 455 & 1085 & $81.2 \pm 4.1$ & $2.4 \pm 0.12$ & $4.1 \times 10^{-3}$ & 613 & 1760 & $213.6 \pm 10.7$ & $2.9 \pm 0.15$ \\
\hline \multirow[t]{4}{*}{ MT5 } & 438 & $3.0 \times 10^{-1}$ & 191 & 324 & $13.1 \pm 0.7$ & $1.7 \pm 0.09$ & $1.2 \times 10^{-2}$ & 332 & 734 & $67.3 \pm 3.4$ & $2.2 \pm 0.11$ \\
\hline & & $1.6 \times 10^{-1}$ & 223 & 409 & $20.9 \pm 1$ & $1.8 \pm 0.09$ & $9.1 \times 10^{-4}$ & 371 & 849 & $90.1 \pm 4.5$ & $2.3 \pm 0.12$ \\
\hline & & $7.6 \times 10^{-2}$ & 266 & 499 & $31.1 \pm 1.6$ & $1.9 \pm 0.1$ & $1.5 \times 10^{-4}$ & 371 & 839 & $88 \pm 4.4$ & $2.3 \pm 0.12$ \\
\hline & & $3.4 \times 10^{-2}$ & 294 & 629 & $49.5 \pm 2.5$ & $2.1 \pm 0.11$ & & & & & \\
\hline \multirow[t]{5}{*}{ MT3 } & 747 & $4.9 \times 10^{-1}$ & 243 & 370 & $10.1 \pm 0.5$ & $1.5 \pm 0.08$ & $9.2 \times 10^{-3}$ & 517 & 1295 & $124.2 \pm 6.2$ & $2.5 \pm 0.13$ \\
\hline & & $3.0 \times 10^{-1}$ & 276 & 455 & $15.3 \pm 0.8$ & $1.6 \pm 0.08$ & $2.8 \times 10^{-3}$ & 553 & 1340 & $133 \pm 6.7$ & $2.4 \pm 0.12$ \\
\hline & & $1.2 \times 10^{-1}$ & 349 & 620 & $28.5 \pm 1.4$ & $1.8 \pm 0.09$ & $5.7 \times 10^{-4}$ & 575 & 1365 & $138 \pm 6.9$ & $2.4 \pm 0.12$ \\
\hline & & $5.2 \times 10^{-2}$ & 405 & 785 & $45.6 \pm 2.3$ & $1.9 \pm 0.1$ & $9.1 \times 10^{-5}$ & 575 & 1385 & $142.1 \pm 7.1$ & $2.4 \pm 0.12$ \\
\hline & & $2.0 \times 10^{-2}$ & 475 & 1060 & $83.2 \pm 4.2$ & $2.2 \pm 0.11$ & & & & & \\
\hline \multirow[t]{4}{*}{ MT6 } & 612 & $3.0 \times 10^{-1}$ & 241 & 405 & $15.6 \pm 0.8$ & $1.7 \pm 0.09$ & $5.4 \times 10^{-3}$ & 455 & 1135 & $122.7 \pm 6.1$ & $2.5 \pm 0.13$ \\
\hline & & $1.2 \times 10^{-1}$ & 303 & 555 & $29.3 \pm 1.5$ & $1.8 \pm 0.09$ & $9.2 \times 10^{-4}$ & 485 & 1145 & $124.9 \pm 6.2$ & $2.4 \pm 0.12$ \\
\hline & & $5.6 \times 10^{-2}$ & 363 & 695 & $46 \pm 2.3$ & $1.9 \pm 0.1$ & $1.1 \times 10^{-4}$ & 485 & 1155 & $127.1 \pm 6.4$ & $2.4 \pm 0.12$ \\
\hline & & $2.0 \times 10^{-2}$ & 406 & 995 & $94.3 \pm 4.7$ & $2.5 \pm 0.13$ & & & & & \\
\hline
\end{tabular}

${ }^{a}$ The radius of gyration of free NaPSS chains, $R_{g}$, was calculated on the basis of the studies in refs 48 and 51 by considering that both the counterions and the added salt contribute the ionic strength. ${ }^{b}$ Some of the $L_{0}$ values are extracted from ref 31 .

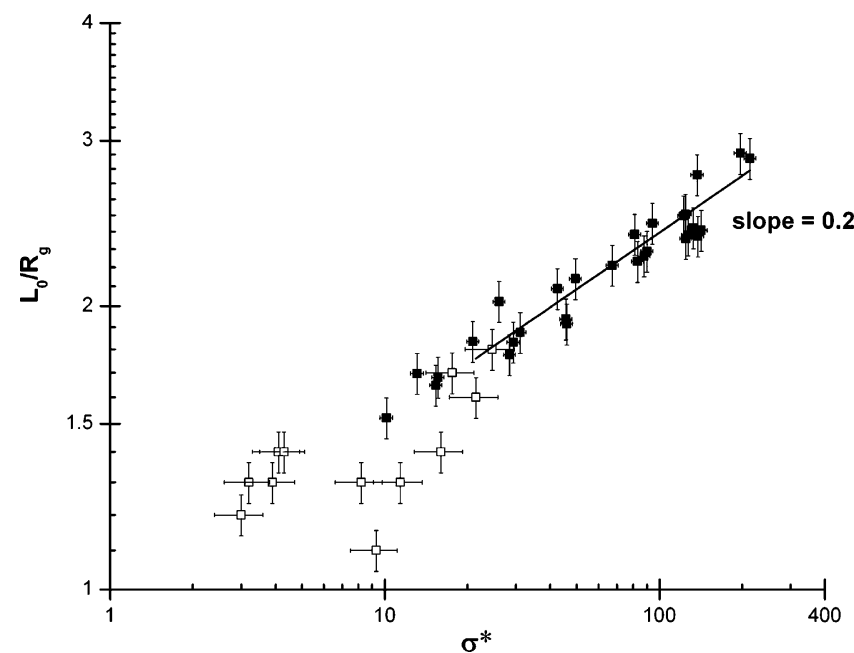

Figure 6. Degree of stretching of the chains as a function of the degree of overlap in a $\log -\log$ plot. Open squares correspond to results from adsorption onto the bare mica surfaces, whereas filled squares are the results from adsorption onto the OTE-modified mica surfaces. The solid line shows the slope of the filled squares.

It is worthwhile to examine how the height of the adsorbed layers changes with added salt concentration. As the salt concentration increases, the sparsely tethered polyelectrolyte chains transit from ordered sticks to salted coils, ${ }^{47}$ the scaling theory predicts that, $L_{0} \propto N^{3 / 5} \sigma^{\circ} C_{\mathrm{s}}^{-1 / 5}$, when the added salt concentration, $C_{\mathrm{s}}$, is greater than the uncondensed counterion concentration in the adsorbed layer $C^{*}$, with $C^{*}$ given by

$$
C^{*}=\frac{N \alpha}{A L_{0}}=\frac{N \sigma \alpha}{L_{0}}
$$

and $L_{0}$ shows no dependence on $C_{\mathrm{s}}$ and $\sigma$ in the limit of low salt concentration $\left(C_{\mathrm{s}}<C^{*}\right){ }^{47}$ If only a minimal amount of intermolecular stretching takes place, a plot of $L_{0} N^{-3 / 5}$ versus $C_{\mathrm{s}}$ should have a slope of $-1 / 5$ in the high-salt limit. Figure 8 shows a $\log -\log$ plot of $L_{0} N^{-v}$ versus $C_{\mathrm{s}}$. The value of $v$ was set to 0.7 for PtBS-NaPSS diblock copolymers adsorbed on the bare mica surfaces and 1 for on the OTE-modified mica surface, respectively, on the basis of the fit of the data in Figure 7. When $C_{\mathrm{s}}<C^{*}$, the height of the layers was found to be independent of the added salt concentration in agreement with the description of Borisov et al. ${ }^{47}$ for the ordered sticks or osmotic brush regime. When $C_{\mathrm{s}}$

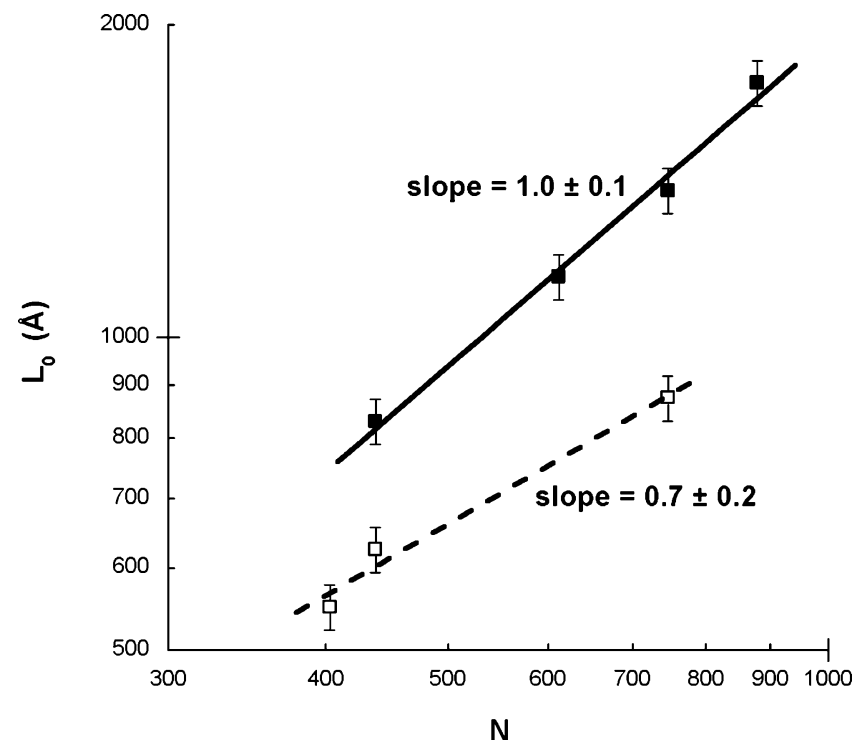

Figure 7. Dependence of the polyelectrolyte layer height, $L_{0}$, on degree of polymerization, $N$, in the low-salt limit for adsorption onto the bare mica surface (open squares) and the OTE-modified mica surface (filled squares). In the low-salt-limit regime, $L_{0}$ is independent of added salt. The straight lines (solid and dashed) are linear fittings for the two cases.

$>C^{*}$, by fitting the points in the high-salt limit, the layer thickness was found to decrease with added salt with a slope of -0.17 for the layer on the bare mice surface and -0.33 for the case of OTE-modified surface, respectively. This suggests that, only when adsorption takes place onto the bare mica surface, the behavior is in agreement with the scaling prediction for the salted coils (sparsely tethered regime), i.e., $L_{0} \propto C_{\mathrm{s}}^{-1 / 5}$.

The height of the adsorbed layers can also be scaled with added salt concentration using the brush model (densely tethered regime). The scaling theory of the brush model suggests that $L_{0}$ $\propto N \sigma^{1 / 3} \mathrm{C}_{\mathrm{s}}{ }^{-1 / 3}$ when $C_{\mathrm{s}}$ is greater than $C^{*}$, and $L_{0}$ does not depend on $C_{\mathrm{s}}$ and $\sigma$ in the limit of low salt $\left(C_{\mathrm{s}}<C^{*}\right) .{ }^{47}$ Therefore, if intermolecular stretching dominates in the self-assembled layers, a plot of $L_{0} N^{-1}$ versus $C_{\mathrm{s}} / \sigma$ should have a slope of $-1 / 3$. Figure 9 shows a plot of $L_{0} N^{-\mathrm{v}}$ versus $C_{\mathrm{s}} / \sigma$, where the value of $v$ was set to 0.7 for polyelectrolytes on the bare mica surface and 1 for on the OTE-modified mica, respectively, on the basis of the fit of the data in Figure 7. In case of the OTE-modified mica surface as the substrate, the results are in excellent accordance with the 


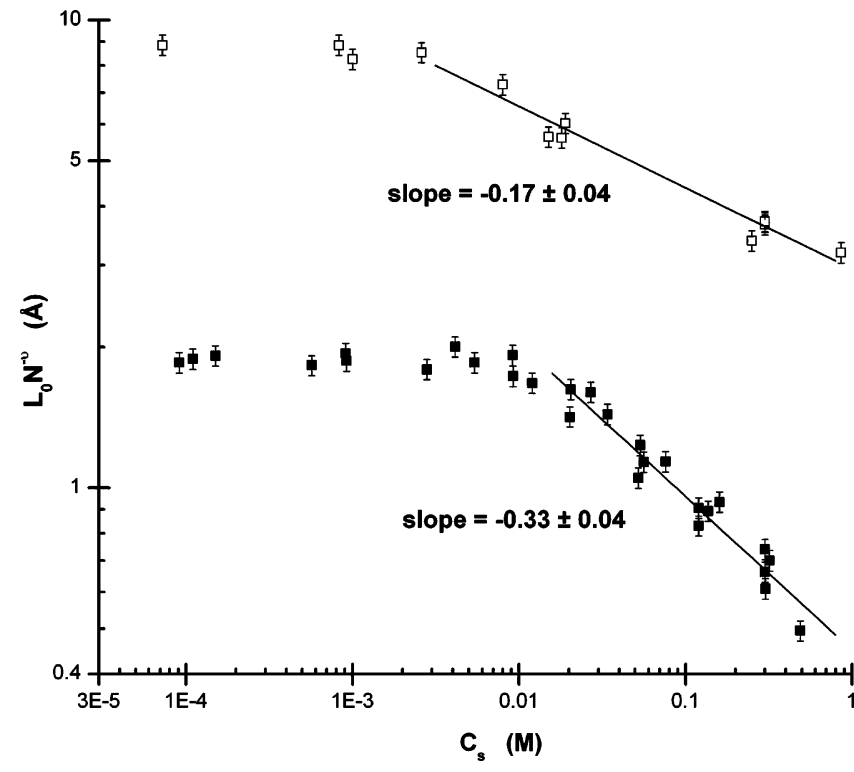

Figure 8. Dependence of the height of the tethered polyelectrolyte layers on the added salt concentration by using the sparse tethering model. Polyelectrolyte was tethered to the bare mica surface (open squares, $v$ was set equal to 0.7 ) and the OTE-modified mica (filled squares, $v$ was set equal to 1 ), respectively. Solid lines are linear fittings of the data points in the high-salt limit. The bare mica case appears to obey the predicted scaling exponent $(-1 / 5)$ of this model, while the OTE-modified mica case does not.

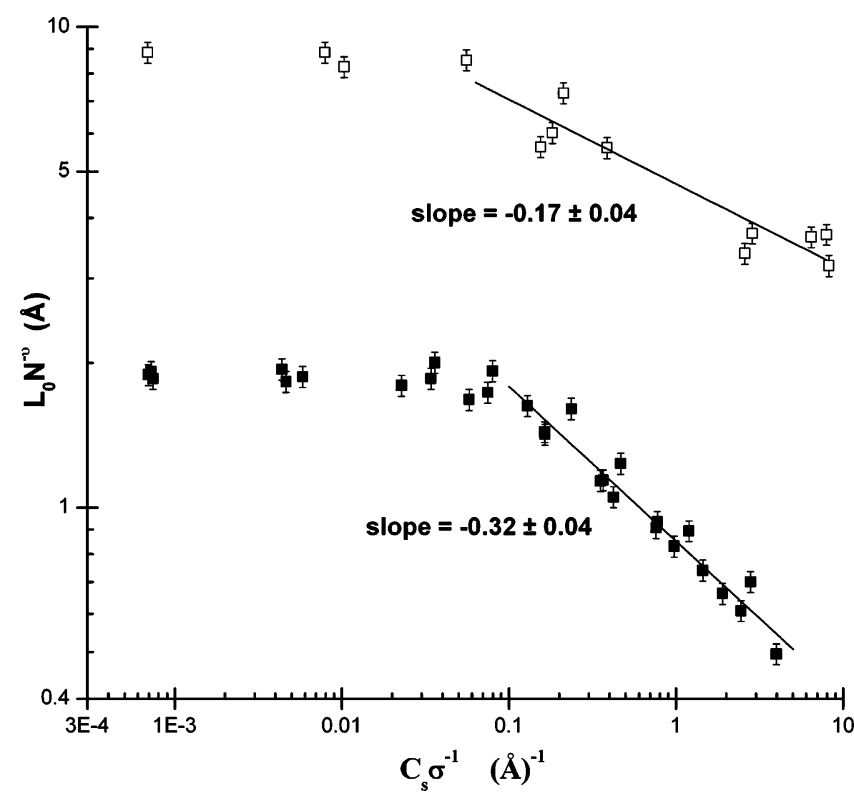

Figure 9. Dependence of the height of the tethered polyelectrolyte layers on the added salt concentration by using the brush model (densely tethering). Polyelectrolyte was tethered to the bare mica surface (open squares, $v$ was set equal to 0.7 ) and the OTE-modified mica (filled squares, $v$ was set equal to 1 ), respectively. Solid lines are linear fittings of the data points in the high-salt limit. The OTEmodified mica case appears to obey the predicted scaling exponent $(-1 / 3)$ of this model, while the bare mica case does not.

scaling predictions for the brush model, with a slope of -0.32 . For the bare mica case, when $C_{\mathrm{s}}>\mathrm{C}^{*}$, the layer thickness was found to decrease with added salt with a slope of -0.17 , which indicates the polyelectrolyte chains are not densely tethered.

Clearly, the sparsely tethered model illustrated in Figure 8 provides a better description of the experimental data resulting from adsorption on the bare mica surface $\left(L_{0} N^{-0.7} \propto C_{\mathrm{s}}{ }^{-0.17}\right)$, whereas the brush structure illustrated in Figure 9 is in excellent
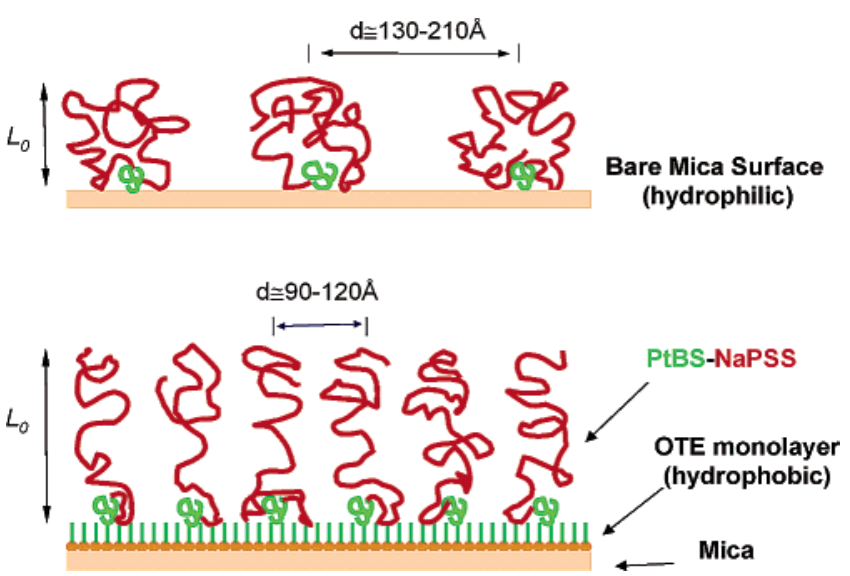

Figure 10. Schematic of the polyelectrolyte layer tethered to the bare mica and the OTE-modified mica surface. A sparse tethering structure is formed when PtBS-NaPSS chains are adsorbed on the bare mica surface, whereas the presence of the hydrophobic OTE layer increases the tethering density such that the chains adopt a brush structure.

agreement with our results obtained from adsorption on the OTEmodified mica surface $\left(L_{0} N^{-1} \propto\left(C_{\mathrm{S}} / \sigma\right)^{-0.32}\right)$. This finding is in line with the previous observations of $L_{0} \propto N^{0.7}$ for the bare mica case and $L_{0} \propto N^{1}$ for the OTE-modified mica case. Taken together, this evidence strongly suggests that the adsorption of the PtBSNaPSS chains to the bare mica surface leads to relatively sparse tethering of the chains, whereas a brush structure is formed through adsorption to the OTE-modified mica surface (see the schematic in Figure 10). The value of $\sigma^{*}$ necessary to ensure enough lateral overlap and to drive additional stretching of the chains seems to be around 10. This means that the chains start to overlap when $L_{0} \cong 3 d$, with $d$ being the average distance between tethered chains and $d^{2}=A$ being the average area per chain. Therefore, the tethered polyelectrolyte chains can stretch as much as three times the average lateral distance before the chains start to overlap.

\section{Conclusion}

OTE molecules were deposited to the mica surface by using the LB technique. AFM imaging showed that the distribution of the OTE monolayer was homogeneous, rather than the reported island morphology. ${ }^{33,}{ }^{34}$ Hydrophobically modified polyelectrolytes were adsorbed from aqueous salty solutions to both the bare mica surface and the OTE-modified mica surface. Surface force experiments and ellipsometric measurements showed that polyelectrolytes tethered to the OTE-modified mica surface are dense enough to form a brush structure and their behavior can be well described by the scaling brush model; whereas for polyelectrolytes tethered to the bare mica surface, the chains adopt a sparse tethering structure. In the low-salt limit, the height of the sparsely tethered chains scales with the degree of polymerization as $L_{0} \propto N^{0.7} . L_{0} N^{-0.7}$ does not depend the added salt concentration in the low-salt regime and scales with the salt concentration to the -0.17 power in the high-salt regime, in agreement with the scaling prediction for sparsely tethered polyelectrolyte chains.

Acknowledgment. The authors wish to thank Ryan Toomey and Markus Biesalski for helpful discussions. This work was financially supported by NSF Grant No. CTS-9616797 from the CTS and DMR Divisions of the National Science Foundation, by the MRSEC and NIRT programs of the National Science Foundation under Awards No. DMR00-80034 and CTS01-03516, and by the Institut Français du Pétrole (I.F.P., France).

LA052293L 